\title{
THE RELATION BETWEEN CONTINUITY AND DIFFERENTIABILITY OF FUNCTIONS ON ALGEBRAS
}

\author{
R. F. RINEHART ${ }^{1}$ AND JACK C. WILSON
}

1. Introduction. Let $\mathfrak{A}$ be a finite dimensional associative algebra with an identity over the real or complex field $\mathfrak{F}$, and let $f$ be a function on $\mathfrak{A}$ to $\mathfrak{A}$, i.e., a function with domain and range in $\mathfrak{A} . \mathfrak{A}$ is a normable ring, hence a metric topological space in the metric induced by the chosen norm [4]. Consequently, the usual elementary concepts of limits and continuity make sense, and the customary elementary theorems are valid.

In [2] a generalized difference quotient definition of differentiability and derivative was given for a function $f$ for the case of a total matrix algebra over $\mathfrak{F}$. This definition, which is equally applicable to any finite dimensional associative algebra $\mathfrak{A}$ over $\mathfrak{F}$, is:

Definition. Let $f(\xi)$ be a function with domain and range in $\mathfrak{A}$ defined in some neighborhood of $\xi=\alpha$. Then $f(\xi)$ is said to be differentiable at $\xi=\alpha$, if, for all $\delta \in \mathfrak{A}$ in a sufficiently small neighborhood $\mathfrak{N}$ of 0 ,

(I) the difference $f(\alpha+\delta)-f(\alpha)$ is expressible as a finite sum of the form $f(\alpha+\delta)-f(\alpha)=\sum_{i=1}^{k} \lambda_{i} \delta \mu_{i}$ where $\lambda_{i}, \mu_{i} \in \mathfrak{A}$, and

(II) $\lim _{\delta \rightarrow 0} \sum_{i=1}^{k} \lambda_{i} \mu_{i}$ exists.

If I and II are fulfilled, then the limit in II is called the derivative of $f(\xi)$ at $\xi=\alpha$, and is denoted by $f^{I}(\alpha)$.

If $\mathfrak{A}$ is commutative, it is easily verified that the above definition implies that $f(\xi)$ is Fréchet-differentiable at $\xi=\alpha$, which in turn implies that $f(\xi)$ is continuous at $\xi=\alpha[1]$. For noncommutative $\mathfrak{A}$ this inference is not warranted.

The proofs in [2] of the uniqueness of the derivative, and of the theorems concerning differentiability and derivative of the sum or product of two functions, are equally valid for the more general algebras $\mathfrak{A}$ of the type considered here. However, the proof that the product $f g$ of two functions is differentiable at $\xi=\alpha$ if $f$ and $g$ are, and that $(f g)^{I}(\alpha)=f^{I}(\alpha) g(\alpha)+f(\alpha) g^{I}(\alpha)$, assumed that at least one of the functions $f, g$ was continuous at $\xi=\alpha$, and it was conjectured that (A) this hypothesis is essential since (B) differentiability at a point does not imply continuity at the point.

Presented to the Society, January 23, 1962; received by the editors January 23, 1962.

1 Supported by ONR grant. 
In this note the above double conjecture is settled for any $\mathfrak{A}$ as defined above by disproving Conjecture A, and proving Conjecture B, together with some theorems which get at the essential reason for the validity of $B$ and settle a question of N. J. Fine.

2. Differentiability of the product of differentiable functions. As a preliminary to the treatment of Conjecture $A$, the definition of differentiability is recast as follows.

Lemma 2.1. A function $f(\xi)$ defined in a neighborhood of $\xi=\alpha \in \mathfrak{A}$, is differentiable at $\xi=\alpha$ if and only if, for all $\delta \in \mathfrak{A}$ with sufficiently small norm, the functional difference can be written in the form

$$
f(\alpha+\delta)-f(\alpha)=\delta \Phi+\sum_{i=1}^{m} \nu_{i} \delta \eta_{i}, \quad\left(\Phi, \nu_{i}, \eta_{i} \in \mathfrak{U}\right)
$$

where $\lim _{\delta \rightarrow 0} \Phi$ exists and

$$
\sum_{i=1}^{m} \nu_{i} \eta_{i}=0
$$

Proof. The sufficiency of the condition follows directly from the definition. For the necessity, we observe that from condition I of the definition, for $\|\delta\|$ sufficiently small, $f(\alpha+\delta)-f(\alpha)=\delta \sum_{i=1}^{k} \lambda_{i} \mu_{i}$ $+\left[\sum_{i=1}^{k} \lambda_{i} \delta \mu_{i}-\delta \sum_{i=1}^{k} \lambda_{i} \mu_{i}\right]$. Since $\mathfrak{A}$ has an identity, the bracketed expression is of the form $\sum_{i=1}^{m} \nu_{i} \delta \eta_{i}$. Further $\sum_{i=1}^{m} \nu_{i} \eta_{i}=0$, and condition II of the definition assures that $\lim _{\delta \rightarrow 0} \sum_{i=1}^{k} \lambda_{i} \mu_{i}$ exists.

Conjecture $A$ is now settled in the negative by

THEOREM 2.1. Let $f(\xi)$ and $g(\xi)$ be functions differentiable at $\xi=\alpha \in \mathfrak{R}$. Then $p(\xi)=f(\xi) g(\xi)$ is differentiable at $\xi=\alpha$ and $p^{I}(\alpha)=f^{I}(\alpha) g(\alpha)$ $+f(\alpha) g^{I}(\alpha)$.

Proof. By the lemma

$$
\begin{aligned}
& f(\alpha+\delta)=f(\alpha)+\delta \Phi+\sum_{i=1}^{k} \nu_{i} \delta \eta_{i} \\
& g(\alpha+\delta)=g(\alpha)+\delta \Psi+\sum_{i=1}^{m} \theta_{i} \delta \pi_{i}
\end{aligned}
$$

such that $\lim _{\delta \rightarrow 0} \Phi=f^{I}(\alpha), \lim _{\delta \rightarrow 0} \Psi=g^{I}(\alpha)$, and

$$
\sum_{i=1}^{k} \nu_{i} \eta_{i}=\sum_{i=1}^{m} \theta_{i} \pi_{i}=0 \text { for all } \delta \in \mathfrak{A} \text { in some neighborhood of } 0 \text {. }
$$


Therefore,

$$
\begin{aligned}
p(\alpha+\delta)-p(\alpha)= & f(\alpha+\delta) g(\alpha+\delta)-f(\alpha) g(\alpha) \\
= & {\left[f(\alpha)+\delta \Phi+\sum_{i=1}^{k} \nu_{i} \delta \eta_{i}\right]\left[g(\alpha)+\delta \Psi+\sum_{i=1}^{m} \theta_{i} \delta \pi_{i}\right] } \\
& -f(\alpha) g(\alpha) \\
= & f(\alpha) \delta \Psi+f(\alpha) \sum_{i=1}^{m} \theta_{i} \delta \pi_{i}+\delta \Phi g(\alpha)+\delta \Phi \delta \Psi \\
& +\delta \Phi \sum_{i=1}^{m} \theta_{i} \delta \pi_{i}+\left[\sum_{i=1}^{k} \nu_{i} \delta \eta_{i}\right] \delta \Psi \\
& +\left[\sum_{i=1}^{k} \nu_{i} \delta \eta_{i}\right] g(\alpha)+\left[\sum_{i=1}^{k} \nu_{i} \delta \eta_{i}\right]\left[\sum_{i=1}^{m} \theta_{i} \delta \pi_{i}\right] .
\end{aligned}
$$

Thus the difference $p(\alpha+\delta)-p(\alpha)$ satisfies condition I of the definition of differentiability. To verify condition II, we need to establish the existence of the limit of a "detached coefficient" of $\delta$. Any such coefficient will do, by virtue of the uniqueness of the derivative, when it exists [2]. One such "detached coefficient" is,

$$
\begin{aligned}
f(\alpha) \Psi+\Phi g(\alpha)+\delta \Phi \Psi+f(\alpha) & \sum_{i=1}^{m} \theta_{i} \pi_{i}+\delta \Phi \sum_{i=1}^{m} \theta_{i} \pi_{i}+\left[\sum_{i=1}^{k} \nu_{i} \eta_{i}\right] \delta \Psi \\
+ & {\left[\sum_{i=1}^{k} \nu_{i} \eta_{i}\right] g(\alpha)+\left[\sum_{i=1}^{k} \nu_{i} \eta_{i}\right]\left[\sum_{i=1}^{m} \theta_{i} \delta \pi_{i}\right] }
\end{aligned}
$$

which by equations 2.1 , is

$$
f(\alpha) \Psi+\Phi g(\alpha)+\delta \Phi \Psi \text {. }
$$

The limit as $\delta \rightarrow 0$ of this expression exists, since the limits of $\Phi$ and $\Psi$ exist, and that limit is $f(\alpha) g^{I}(\alpha)+f^{I}(\alpha) g(\alpha)$.

3. Differentiability does not imply continuity. Theorem 2.1 was proved without invoking continuity of $f$ or $g$ at $\alpha$ as in [2]. Indeed, the following example, communicated by N. J. Fine, shows that a function may be differentiable at $\alpha \in \mathfrak{P}$ and discontinuous at $\alpha$.

Let $\mathfrak{A}$ be the algebra of $2 \times 2$ matrices over the real field. For

$$
\xi=\left(\begin{array}{ll}
x_{11} & x_{12} \\
x_{21} & x_{22}
\end{array}\right)
$$

let $M_{\xi}=\max _{i, j}\left|x_{i j}\right|$. Define a function $f(\xi)$ by, 


$$
\begin{aligned}
& f(\xi)=\left(\begin{array}{cc}
x_{11} & M_{\xi}^{2} x_{12} \\
x_{21} / M_{\xi}^{2} & x_{22}
\end{array}\right), \quad \text { for } \xi \neq 0 \\
& f(0)=0 .
\end{aligned}
$$

$f(\xi)$ is discontinuous at $\xi=0$, since $\lim _{\xi \rightarrow 0} f(\xi)$ does not exist. However,

$$
f(0+\delta)-f(0)=f(\delta)=P^{-1} \delta P
$$

where

$$
\begin{aligned}
& P=\left(\begin{array}{cc}
M_{\delta} & 0 \\
0 & 1 / M_{\delta}
\end{array}\right) \quad \text { for } \delta \neq 0, \\
& P=I, \text { the identity matrix, for } \delta=0 .
\end{aligned}
$$

Hence $f$ fulfills condition I for differentiability at $\xi=0$. Further $\lim _{\delta \rightarrow 0} P P^{-1}=I$. Hence $f(\xi)$ is differentiable at $\xi=0$.

The example is, so to speak, a pointwise example, and Fine, in the cited communication, raised the question: Is a function which is differentiable in a neighborhood of $\alpha \in \mathfrak{A}$, necessarily continuous at $\alpha$ ? That this is not the case, and why it is not, is shown by the following theorem.

THEOREM 3.1. Let $\epsilon_{1}, \cdots, \epsilon_{n}$ be a basis for $\mathfrak{A}$, with $\epsilon_{1}$ the identity of 2. With $\xi=\sum_{i=1}^{n} x_{i} \epsilon_{i}, \quad x_{i} \in \mathfrak{F}$, let $f(\xi)=\sum_{i=1}^{n} f_{i}\left(x_{1}, \cdots, x_{n}\right) \epsilon_{i}$, $f_{i}\left(x_{1}, \cdots, x_{n}\right) \in \mathfrak{F}$, be defined in a neighborhood $\mathfrak{x}$ of $\alpha=\sum_{i=1}^{n} a_{i} \epsilon_{i}$, $a_{i} \in \mathfrak{F}$. $A$ necessary condition that $f(\xi)$ be differentiable at $\xi=\alpha$ is that $\partial f_{i} / \partial x_{1}$ exist at $\xi=\alpha$ for $i=1, \cdots, n$. In this case

$$
\left.f^{I}(\alpha)=\sum_{i=1}^{n} \frac{\partial f_{i}}{\partial x_{1}}\right]_{\xi-\alpha} \epsilon_{i} .
$$

Further, if $\mathfrak{A}$ is normal simple over $\mathfrak{F}$, then this condition is also sufficient.

Proof. (a) Necessity. Since $f(\xi)$ is differentiable at $\xi=\alpha$,

$$
f(\alpha+\delta)-f(\alpha)=\sum_{i=1}^{k} \lambda_{i} \delta \mu_{i}
$$

and $\lim _{\delta \rightarrow 0} \sum_{t=1}^{k} \lambda_{i} \mu_{i}$ exists. Choose $\delta=d \epsilon_{1}, d \in \mathfrak{F}$. Then

$$
\lim _{d \rightarrow 0}\left[f\left(\alpha+d \epsilon_{1}\right)-f(\alpha)\right] / d=\lim _{\alpha \rightarrow 0} \sum_{i=1}^{k} \lambda_{i} \mu_{i}=f^{I}(\alpha)
$$

exists. This implies that the limit of each basis component exists, i.e., 


$$
\left.\lim _{d \rightarrow 0} \frac{f_{i}\left(a_{1}+d, a_{2}, \cdots, a_{n}\right)-f_{i}\left(a_{1}, a_{2}, \cdots, a_{n}\right)}{d}=\frac{\partial f_{i}}{\partial x_{1}}\right]_{\xi=\alpha}
$$

exists. Hence

$$
\left.f^{I}(\alpha)=\sum_{i=1}^{n} \frac{\partial f_{i}}{\partial x_{1}}\right]_{\xi=\alpha} \epsilon_{i} .
$$

(b) Sufficiency, under the added hypothesis that $\mathfrak{A}$ is normal simple. Let $\delta=\sum_{i=1}^{n} d_{i} \epsilon_{i}, d_{i} \in \mathfrak{F}$, such that $(\alpha+\delta) \in \mathfrak{N}$. We seek to show first that the difference

$$
f(\alpha+\delta)-f(\alpha)=\sum_{i=1}^{n}\left[f_{i}(\alpha+\delta)-f_{i}(\alpha)\right]_{i},
$$

where $f_{i}(\xi)$ means $f_{i}\left(x_{1}, \cdots, x_{n}\right)$, can be written in the form $\sum_{i=1}^{k} \lambda_{i} \delta \mu_{i}$, or expressing $\delta, \lambda_{i}, \mu_{i}$ in terms of the basis elements, that

$$
f(\alpha+\delta)-f(\alpha)=\sum_{i, j=1}^{n} t_{i j} \epsilon_{i}\left(\sum_{k=1}^{n} d_{k} \epsilon_{k}\right) \epsilon_{j} .
$$

If $c_{i j k}$ are the multiplication constants for the basis $\epsilon_{1}, \cdots, \epsilon_{n}$, i.e., $\epsilon_{i} \epsilon_{j}=\sum_{k=1}^{n} c_{i j k} \epsilon_{k}$, then 3.1 can be written

$$
f(\alpha+\delta)-f(\alpha)=\sum_{i, j, k, r, \delta-1}^{n} t_{i j} d_{k} c_{i k s} c_{s j r} \epsilon_{r}
$$

Equation 3.2 will be fulfilled for arbitrary $\delta$ in $\Re$ i.e., arbitrary $d_{k}$, if and only if the system of linear equations over $\mathfrak{F}$,

$$
f_{r}(\alpha+\delta)-f_{r}(\alpha)=\sum_{s, i, j=1}^{n} t_{i j} c_{i k_{s}} c_{s j r}, \quad k, r=1, \cdots, n
$$

is solvable for the coefficients $t_{i j}$. Now $f(\alpha+\delta)-f(\alpha)$ can be written in the form

$$
f(\alpha+\delta)-f(\alpha)=\sum_{r=1}^{n}\left[f_{r}(\alpha+\delta)-f_{r}(\alpha)\right] \epsilon_{r}=\sum_{r=1}^{n}\left(\sum_{k=1}^{n} g_{r k} d_{k}\right) \epsilon_{r}
$$

where

$$
\begin{aligned}
g_{r s}= & {\left[f_{r}\left(a_{1}+d_{1}, \cdots, a_{s}+d_{s}, a_{s+1}, \cdots, a_{n}\right)\right.} \\
& \left.-f\left(a_{1}+d_{1}, \cdots, a_{s-1}+d_{s-1}, a_{s}, \cdots, a_{n}\right)\right] d_{s}^{-1}, \quad \text { if } d_{s} \neq 0, \\
g_{r s}= & 0, \quad \text { if } s \neq 1 \text { and } d_{s}=0, \\
g_{r 1}= & \frac{\partial f_{r}}{\partial x_{1}}, \quad \text { if } d_{1}=0 .
\end{aligned}
$$


Hence the problem of satisfying 3.2 for arbitrary $d_{k}$ is transformed to that of showing that the system of linear equations over $\mathfrak{F}$,

$$
\sum_{s, i, j} t_{i j} c_{i k s} c_{s j r}=g_{r k} \quad(r, k=1, \cdots, n),
$$

is solvable for the $t_{i j}$. Since $\mathfrak{A}$ is normal simple, the coefficient matrix of the $t_{i j}$ is nonsingular [3], and 3.4 has a unique solution for the $t_{i j}$. Hence

$$
\begin{aligned}
f(\alpha+\delta)-f(\alpha) & =\sum_{r, k}^{n} g_{r k} d_{k} \epsilon_{r} \\
& =\sum_{i, j, k, r, s=1}^{n} t_{i j} c_{i k_{s}} c_{s j r} d_{k} \epsilon_{r} \\
& =\sum_{i, j=1}^{n} t_{i j} \epsilon_{i} \delta \epsilon_{j}
\end{aligned}
$$

and the function $f$ fulfills requirement $I$ of the definition for $\alpha+\delta$ in $\Re$.

Now $\lim _{\delta \rightarrow 0} \sum_{i, j=1}^{n} t_{i j} \epsilon_{i} \epsilon_{j}$ exists if and only if the limit of its $r$ th component, $r=1, \cdots, n$, exists. The $r$ th component is $\sum_{i, j=1}^{n} t_{i j} c_{i j r}$ $=\sum_{i, j, \mathrm{k}=1}^{n} t_{i j} c_{i 1 k} c_{k j r}$, since $c_{i 1 k}=1$ if $k=i, 0$ otherwise, and

$$
\begin{aligned}
\lim _{\delta \rightarrow 0} \sum_{i, j} t_{i j} c_{i 1 k} c_{k j r} & =\lim _{\delta \rightarrow 0} g_{r 1}=\lim _{a_{1} \rightarrow 0} g_{r 1} \\
& =\lim _{d_{1} \rightarrow 0} \frac{f_{r}\left(a_{1}+d_{1}, a_{2}, \cdots, a_{n}\right)-f_{r}\left(a_{1}, \cdots, a_{n}\right)}{d_{1}} \\
& \left.=\frac{\partial f_{r}}{\partial x_{1}}\right]_{\xi=\alpha} .
\end{aligned}
$$

This holds for each $r=1, \cdots, n$, hence $f(\xi)$ is differentiable at $\xi=\alpha$.

Theorem 3.1 shows that for a normal simple algebra $\mathfrak{A}$, e.g., a total matrix algebra, differentiability of $f(\xi)$ at $\xi=\alpha$, requires only that the component functions of $f(\xi)$ be differentiable with respect to the identity component of $\xi$; they may be any functions whatever, continuous or not, of $x_{2}, \cdots, x_{n}$, provided only that $f(\xi)$ is defined in $\Re$.

Normal simple algebras are the most general algebras satisfying the second part of Theorem 3.1 in the following sense.

THEOREM 3.2. If $\mathfrak{A}$ is not normal simple, then there exists at least one function on $\mathfrak{A}$ to $\mathfrak{A}$, with analytic component functions, which is not differentiable at any element of $\mathfrak{A}$.

Proof. Suppose $\mathfrak{A}$ is not simple. Then $\mathfrak{A}$ contains a nontrivial twosided ideal $\mathfrak{E}$. The identity $\epsilon_{1}$ of $\mathfrak{A}$ does not belong to $\mathfrak{E}$. Since $\mathfrak{E} \neq 0$, 
$\exists$ an element $\epsilon_{2} \in \mathfrak{C}$ independent of $\epsilon_{1}$ over $\mathfrak{F}$. Then $\mathfrak{A}$ has a basis $\epsilon_{1}, \epsilon_{2}, \cdots, \epsilon_{n}$ including $\epsilon_{1}$ and $\epsilon_{2}$. The function $f(\xi)=x_{2} \epsilon_{1}$ has component functions analytic for all $\xi \in \mathfrak{A}$. If $f(\xi)$ were differentiable at some $\alpha \in \mathfrak{A}$, then for all $\delta$ with $\|\delta\|$ sufficiently small,

$$
f(\alpha+\delta)-f(\alpha)=\sum_{i, j=1}^{n} t_{i j} \epsilon_{i} \delta \epsilon_{j}
$$

Choose $\delta=d_{2} \epsilon_{2} \in \mathfrak{C}$ with $d_{2} \neq 0$ in $\mathfrak{F}$, and $\left|d_{2}\right|$ appropriately small. Then for all such $d_{2}$,

$$
f\left(\alpha+d_{2} \epsilon_{2}\right)-f(\alpha)=d_{2} \epsilon_{1}=\sum_{i, j=1}^{n} t_{i j} \epsilon_{i} d_{2} \epsilon_{2} \epsilon_{j},
$$

whence

$$
\epsilon_{1}=\sum_{i, j=1}^{n} t_{i j} \epsilon_{i} \epsilon_{2} \epsilon_{j}
$$

is in $\mathfrak{E}$, a contradiction. Hence $f(\xi)$ is differentiable at no $\alpha \in \mathfrak{A}$.

Suppose that $\mathfrak{A}$ is not normal. Then $\exists$ an element $\epsilon_{2} \in \mathfrak{A}$, independent of $\epsilon_{1}$, lying in the centrum of $\mathfrak{A}$. Consider again $f(\xi)=x_{2} \epsilon_{1}$ and suppose that $f(\xi)$ is differentiable at $\xi=\alpha$. Choosing $\delta=d_{2} \epsilon_{2}$, then as above

$$
\epsilon_{1}=\sum_{i, j=1}^{n} t_{i j} \epsilon_{i} \epsilon_{2} \epsilon_{j}=\left(\sum_{i, j=1}^{n} t_{i j} \epsilon_{i} \epsilon_{j}\right) \epsilon_{2} .
$$

Since $\lim _{\delta \rightarrow 0} \sum_{i, j=1}^{n} t_{i j} \epsilon_{i} \epsilon_{j}$ exists, 3.5 implies that $\epsilon_{1}=f^{I}(\alpha) \epsilon_{2}$. But by Theorem 3.1, $f^{I}(\alpha)=\partial f /\left.\partial x_{1}\right|_{\xi=\alpha}=0$. Hence $\epsilon_{1}=0$, a contradiction.

\section{RFFERENCES}

1. E. Hille and R. S. Phillips, Functional analysis and semigroups, Amer. Math. Soc. Colloq. Publ. Vol. 30, 2nd ed., Amer. Math. Soc., Providence, R. I., 1957.

2. R. F. Rinehart, Extension of the derivative concept for functions of matrices, Proc. Amer. Math. Soc. 8 (1957), 329-335.

3. K. Shoda, Ein Kriterium für normale einfache hyperkomplexe Systeme, Proc. Imp. Acad. Japan 10 (1934), 195-197.

4. R. W. Wagner, Differentials and analytic continuation in non-commutative algebras, Duke Math. J. 7 (1940), 677-691.

Case Institute of Technology and

Florida Presbyterian College 